\title{
Problemas con las fuentes escritas y su interpretación: De cuestionarios, franciscanos e "indios" en México, siglo XVI
}

JESÚS BUSTAMANTE

Instituto de Historia. CSIC. Madrid

\section{RESUMEN}

El autor se centra en el caso concreto de la obra de Fray Bernardino de Sahagún (México, siglo XVI) para estudiar los límites del método de investigación y sus implicaciones no previstas, atendiendo especialmente al sistema de cuestionarios.

Palabras clave: Sahagún, México, Siglo XVI, Cultura Nahuatl, Cuestionarios, Método antropológico.

\section{SUMMARY}

The author deals with the case of Fray Bernardino de Sahagún's work (XVth Century Mexico) to study the limits of research method and its unforseen implications, paying a special attention to the system of questionnairies.

Key words: Sahagún, Mexico, XVI ${ }^{\text {th }}$ Century, Nahuatl Culture, Questionnairies, Anthropological Method.

Para identificar adecuadamente el problema ${ }^{1}$ o, mejor dicho, la problemática sobre la que me gustaría reflexionar, nada me parece más oportuno que empezar exponiendo una anécdota personal que considero harto significativa. Se refiere a los primeros años de mi carrera profesional, más aún corresponde a la primera investigación de cierta envergadura a la que me he enfrentado.

Recién terminada la carrera de Antropología Americana en la Universidad Complutense; con los textos, teorías y debates más recientes bullendo en mi cabeza; y con una declarada vocación por el México

${ }^{1}$ Este trabajo se inscribe en el proyecto de investigación número BSO2001-2341 del Plan Nacional de Investigación del Ministerio de Ciencia y Tecnología.

RDTP, LVIII, 1 (2003): 221-236 
prehispánico que ya para entonces me había hecho iniciar el estudio de la lengua náhuatl, mi objetivo era entoces ofrecer Una aproximación al sistema político mexica ${ }^{2}$. Para ello, comencé elaborando un modelo teórico que daba un buen repaso crítico a las propuestas de la Antropología Política del momento para concretarse después en unos planteamientos precisos que se derivaban en lo esencial de las aportaciones de la Ciencia Política y, en especial, de las que un politólogo - Ronald Cohenhabía hecho en estrecho diálogo-debate con la Antropología. Armado con ese instrumento intelectual tan elaborado (de hecho era absurdamente extenso y detallado) pasé a su aplicación a los materiales documentales disponibles.

Y entre esos materiales el primer lugar le correspondía, por supuesto, a los textos en lengua náhuatl, aquéllos en los que los indígenas describían su propio sistema cultural, social y político en su propio idioma. Y había que empezar por la fuente de las fuentes, la autoridad incuestionada y última en lo que respecta al México central prehispánico de lengua náhuatl: fray Bernardino de Sahagún y su extensísima obra enciclopédica sobre los antiguos mexicanos, la llamada Historia General -o Universalde las cosas de la Nueva España ${ }^{3}$. Una obra verdaderamente extraordinaria, cuya veracidad y neutralidad se han enfatizado hasta tal extremo que sus textos han llegado a ser definidos como obra de los informantes de Sahagún (no del franciscano) y sus contenidos como la voz prístina - aún sin contaminar- de los indígenas anteriores a la conquista española. Volveremos más tarde sobre estas afirmaciones.

En mi caso concreto la obra de Sahagún era doblemente importante, no sólo porque proporciona un vocabulario indígena muy rico, además de textos más o menos primarios sobre el tema, sino porque esos textos constituyen de hecho un todo coherente y fuertemente articulado que Sahagún clasificó como "Libro VIII", dedicado enteramente al señorío indígena y sus formas de gobierno. Más aún, la forma en que el material etnográfico es presentado en ese libro unitario facilita notablemente am-

\footnotetext{
2 Así se tituló la tesis de licenciatura, leída en 1981.

3 Dada la complejidad de la obra de Sahagún es difícil remitir a una edición en concreto. Cabe decir que la única versión integrada del texto náhuatl, texto castellano e ilustraciones corresponde a la edición facsimilar del Códice Florentino (Sahagún 1979). La única donde puede encontrarse el texto en lengua náhuatl completo, con una traducción al inglés, es la publicada por Arthur J. O. Anderson y Charles E. Dibble (Sahagún 1950-1980). Y la única que edita el texto castellano original del Códice Florentino, sin mediaciones y tareas intermedias de edición por haberse suprimido fragmentos, referencias, etc., es la publicada por Alfredo López Austin y Josefina García Quintana (Sahagún 1988).
} 
pliarlo e ilustrarlo con otro tipo de fuentes indígenas de primer orden, como el Códice Mendoza por ejemplo, y todo ello a su vez resultaba muy fácil de interpretar y ajustar en un solo modelo político de notable coherencia y simplicidad. Los resultados llegaron a ser tan espectaculares, sobre todo en lo que se refería a la articulación institucional y administrativa, que empecé a sospechar que algo raro estaba ocurriendo. El modelo que iba surgiendo del análisis no sólo era coherente, sino que por alguna razón me resultaba sorprendentemente familiar. Tras alguna reflexión y cierta indagación pude constatar, con auténtico espanto, que aquella pormenorizada restitución que yo había hecho del sistema político mexica anterior a la llegada de los españoles era un reflejo casi literal del modelo político y, sobre todo, administrativo de la Castilla de finales del siglo XV y principios del XVI; como si la larga mano de los Reyes Católicos también hubiera llegado al México prehispánico del último Moctezuma. Al mismo tiempo me dí cuenta de que mi modelo tenía además clarísimas deficiencias, pues en él no había lugar $\longrightarrow$ no un lugar suficiente- para figuras sin duda esenciales del antiguo sistema prehispánico, como el Huei Tlabtoani (es decir, nada menos que el puesto que ocupaba el propio Moteuczoma) o el Huei Cibuacoatl (el segundo puesto en importancia política, una especie de alter-ego del anterior pero cuyo papel hasta el día de hoy no entendemos con precisión).

Al margen de otro tipo de consideraciones que ahora no son pertinentes, podía decirse que mi método de análisis textual había sido un éxito completo pero en un sentido contrario al que yo pretendía. De la fuente mexicana por antonomasia había surgido un retrato fiel de la sociedad castellana. En otras palabras, el método aplicado me permitió aislar con auténtica precisión el reflejo que el autor europeo había proyectado de sí mismo sobre su obra, pero había oscurecido por completo el retrato que ese mismo autor había pretendido hacer de la sociedad mexica, a pesar de haberlo hecho recurriendo al vocabulario y a la forma de pensar nahuas.

Tenemos aquí planteado pues - y con toda claridad - uno de los problemas que yo considero más apasionantes de los que afectan a los textos y en general a las fuentes escritas, cualquiera que sea su condición y cualquiera que sea la fidelidad o la neutralidad que les concedamos. Pero a mí, por aquellos entonces, más que filosófica admiración o reflexión hermenéutica, lo que me produjo todo aquello fue un auténtico quebradero de cabeza porque a partir de ese punto resultaba muy poco convincente seguir adelante con el dichoso modelo político mexica. Procedí pues a resolver la cuestión como mejor pude, obviando las purezas metodológicas y recurriendo al sanísimo y socorrido "eclecticismo" de 
fuentes, métodos y de todo lo que hiciera falta. El resultado final no fue malo pero, ciertamente, no se ajustaba a mi proyecto inicial y se había alcanzado recurriendo a lo que yo consideraba trampas conceptuales y metodológicas manifiestas, razón por la cual nunca lo publiqué.

En todo caso, aunque aquella investigación se terminara y cumpliera sus objetivos académicos, el problema que había planteado siguió en mi cabeza por muchos años. De hecho fue el punto de partida para mi tesis doctoral, titulada precisamente La obra etnográfica y lingüística de fray Bernardino de Sahagún ${ }^{4}$. Es decir, tras la extraña experiencia sufrida indagando sobre el sistema político de los antiguos mexicas, mis intereses como investigador se desplazaron necesariamente de las sociedades prehispánicas a las descripciones que sobre esas sociedades se habían hecho durante la colonia. Y sobre algunas de las conclusiones, soluciones y nuevas perplejidades a las que he ido llegando a lo largo de los años siguientes es sobre lo que ahora quisiera hablar, pero antes me parece oportuno dar unas cuantas referencias y precisiones generales, que considero imprescindibles para entendernos mejor.

Bernardino de Sahagún es una destacada personalidad, bien conocida desde hace años, hasta el extremo de que alrededor de su figura y de su obra se ha ido elaborando un bibliografía tan amplia y compleja que hoy los "estudios sahaguntinos" son casi un campo especializado de investigación $n^{5}$. Muy brevemente podemos decir que su vida es larga en años, pues cubre casi todo el siglo XVI (1499-1590), pero relativamente sencilla en su desarrollo. Se puede resumir en que, tras estudiar en la Universidad de Salamanca y hacerse franciscano reformado, marchó a América en 1529 para pasar los restantes sesenta años de su vida como misionero en México. Ahora bien, de esos sesenta años de misión entre los indios de la Nueva España, alrededor de cuarenta los consagró a la enseñanza su-

\footnotetext{
${ }^{4}$ Con ese título fue leída en 1987 y publicada, sin modificaciones y en corta edición, por la propia Universidad Complutense (Bustamante 1989). De ella surgieron después varias publicaciones, especialmente dos: una concentrada en las cuestiones bibliográficas, críticas y documentales (Bustamante 1990) y otra concentrada en la dimensión más interpretativa, así como en los modelos usados por Sahagún (Bustamante 1992).

${ }^{5}$ La mejor guía para adentrarse en ese campo me sigue pareciendo la obra editada por Munro E. Edmonson (1974), completada por la de J. Jorge Klor de Alva, Henri B. Nicholson y Eloise Quiñones Keber (1988). Por supuesto que hay otras muchas más, entre las que yo destacaría, por su dimensión crítica, la de Walden Browne (2000). En lo que se refiere a estudios biográficos propiamente dichos, la monografía más completa sigue siendo la clásica de Luis Nicolau d'Olwer (1952), aunque haya que completarla con referencias posteriores (como las incluidas en mi propio trabajo Bustamante 1990).
} 
perior en ese centro de formación para una nueva élite indígena que fue el Colegio de Santa Cruz de Santiago de Tlatelolco (de donde surgieron precisamente los ayudantes trilingües del franciscano; varios de los cuales ocuparían además importantes puestos políticos). Pero eso no es todo, al menos cuarenta y cinco años los consagró a la investigación y a escribir una obra lingüística y etnográfica sobre las poblaciones nahuas del Centro de México que hoy es un referente obligado para los estudios del México antiguo (especialmente para los llamados "aztecas").

Así pues Sahagún fue un misionero bastante singular al actuar como un especialista retirado por largos años de las tareas más ordinarias; pero la obra que realizó no fue menos paradójica e inusual. Alejada del modelo descriptivo utilizado por otros misioneros de la época, esa obra reúne unas características que la hacen una fuente ideal de materiales etnográficos y lingüísticos, al mismo tiempo que también la hacen difícilmente perceptible como una producción histórica europea dotada necesariamente de objetivos, intenciones, presupuestos y modelos paradigmáticos precisos. Más aún, hasta la figura de Sahagún se vuelve borrosa en ella, oscilando entre los papeles de autor, editor y simple colector de los materiales que ofrece. Nada más peligroso ni más engañoso a la hora de utilizarla, como ya sabemos por la anécdota que acabo de contar. Conviene por tanto que nos detengamos con mayor atención en esas especiales características textuales de la obra de Sahagún.

Ante todo es preciso señalar que el título de Historia Universal de las Cosas de la Nueva España hace referencia no tanto a un escrito específico, como a un complejísimo proyecto de investigación; ese mismo que se desarrolló a lo largo de unos cuarenta años y que, lógicamente, se fue plasmando en un amplio conjunto de manuscritos sucesivos. No voy a entrar en cuestiones críticas, sólo indicar que un largo camino media entre los escritos germinales de 1547 (sólo en náhuatl) y los terminales de 1585 (sólo en castellano y esencialmente pensados para rescatar lo más indispensable del proyecto). Entre medias tenemos lo que yo llamo los "Manuscritos Mayores" y muy especialmente los tres grandes volúmenes en folio del Códice Florentino, realizado entre 1575-77, en el que se contiene el texto bilingüe más completo de la obra (en náhuatl y en castellano), razón por la cual es la versión habitualmente empleada como referencia (aunque es bueno recordar que no es la única, ni tampoco la más compleja).

A pesar de la dificultad que entraña hablar — sin matices - sobre una obra que tiene tantas etapas y tantas versiones diferentes, lo cierto es que la Historia Universal se organiza conceptualmente y se hace materialmente a partir de unos presupuestos metódicos constantes que son los que 
desde el principio del proyecto le dieron una personalidad única y unas características singularísimas. Presupuestos y características que vienen a ser algo así como las claves de su "caja negra", por emplear la expresión de Steve Woolgar ${ }^{6}$. Y a partir de este momento vamos a intentar abrir esa caja negra.

La Historia Universal de Sahagún tiene como primerísima característica el que, siendo la obra de un castellano, ha sido pensada a partir de la lengua indígena. Es decir, su texto básico, aquél que fue ampliándose a lo largo de los años y sobre el que se fueron elaborando o desarrollando todos los demás, es una extensa colección de escritos en lengua náhuatl. Colección diseñada, además, con la finalidad explícita de formar un corpus completo y ejemplar del idioma.

En segundo lugar hay que añadir otra característica que es complementaria de la anterior e igualmente excepcional para la época: esa colección básica de textos en náhuatl fue intencionadamente elaborada -a partir de cuestionarios cuidadosamente dirigidos- para captar el punto de vista indígena. Es decir, esa colección textual en náhuatl tenía por objetivo recoger además la significación ideólogica que los antiguos nahuas daban a sus propias instituciones sociales, su medio natural y su mundo espiritual. En otras palabras, a nuestro fraile no le interesaban tanto las realidades como las ideas que los indígenas tenían de esas realidades, aunque fueran falsas, incorrectas o inexistentes. De nuevo lo que le interesa a Sahagún es el universo lingüístico y cultural, no el universo físico.

Así pues, el texto básico de la Historia Universal (el más valioso para los investigadores actuales) está en lengua náhuatl y, teóricamente al menos, expresa las opiniones y mentalidad indígenas. Pero eso implicaba que las ideas y la personalidad del colector, el español fray Bernardino de Sahagún, debían quedar ocultas detrás de la propia colección textual. $\mathrm{Y}$ esa posición del franciscano tiene un carácter tan metódico e intencionado que incluso se sigue manteniendo en la traducción al castellano que Sahagún elaboró como complemento del texto náhuatl. Las ideas personales del fraile sólo se hicieron explícitas en esos espacios de "autoridad" y de "autoría" que son los "prólogos" y los "apéndices", así como algunas "interpolaciones" que agregó al texto básico (en náhuatl y castellano), añadidos que el franciscano siempre se cuidó de diferenciar con toda claridad. De este modo, rara vez se produce una articulación explícita entre los materiales etnográficos recogidos por Sahagún y sus ideas personales o la forma en que los interpretaba. Este tipo de exposición articulada

${ }^{6}$ Propiamente la afortunada expresión es sólo de la traducción al castellano (Woolgar 1991) y no de la versión inglesa original (Woolgar 1988). 
corresponde más bien a escritos con un carácter muy distinto, como el que representa el Breve compendio de los ritos idolátricos dirigido al Papa Pío $\mathrm{V}$ en $1570^{7}$.

Las características mencionadas hasta ahora son el fundamento por el que Bernardino de Sahagún ha sido considerado como un etnógrafo casi en el sentido moderno, y de que se le haya definido como "pioneer ethnographer of Sixteenth-Century México"8, "padre de la Antropología en el Nuevo Mundo" y otros títulos semejantes. Sin embargo, también conviene señalar que tales características, habitualmente entendidas en un sentido positivo, son también el punto de partida de toda una serie de problemas críticos.

En primer lugar, debido al punto de vista desde el que se escribe la Historia Universal y dada la posición oculta que tiene que asumir su autor, Sahagún tiene muy pocas ocasiones para expresarse personalmente y más raro aún es que mencione las lecturas que hizo o los autores que le sirvieron de inspiración. Se consolida así un estilo retórico original que dota a su obra de una gran frescura y que acentúa todavía más su credibilidad como material etnográfico. Más aún, ese estilo potencia lo novedoso de la Historia Universal al mismo tiempo que parece desarraigarla de cualquier tradición previa europea. Desde un punto de vista retórico es un auténtico hallazgo, pero es conveniente tener en cuenta que se trata exactamente de eso: de un recurso retórico.

Porque el punto de vista indígena desde el que se escribe la obra, unido a ese singular estilo retórico tan refrescante y tan convincente (que yo llamo del "sujeto/autor ausente"), son los que han potenciado la idea de que esos textos son algo ajeno al propio Sahagún. De esa manera, la parte medular de la Historia Universal no se debería al franciscano sino a sus "informantes", que asumirían así la condición de verdaderos "autores", mientras que el fraile pasaría a la condición de puro "colector". Más aún, la radical novedad de la obra, su aparente desarraigo de toda tradición europea y la potencia seductora de su estilo retórico son la causa de que algunos autores llegaran a creer que los "textos de los informantes de Sahagún" eran además la voz prístina y sin mediación alguna de la sociedad y cultura nahuas del Valle de México anterior a la llegada de

\footnotetext{
7 Además de la vieja edición de 1942, existe hoy otra más moderna que incluye la reproducción facsimilar del manuscrito original y su transcripción modernizada (Sahagún 1990).

8 Tal es el subtítulo de la obra editada por Klor de Alva, Nicholson y Quiñones Keber (1988).

9 Así lo designa, por ejemplo, Miguel León-Portilla (1987: 7).
} 
los españoles. Un extremo a todas luces exagerado e insostenible, que sin embargo —quizá por su fuerte componente romántico- se mantuvo por largos años entre los estudiosos más notables de la escuela mexicana y todavía hoy sigue siendo una idea popular fuertemente arraigada ${ }^{10}$.

Hemos llegado así a la situación tal como más o menos estaba cuando yo comencé mi primera investigación. Y tal como demuestra la anécdota personal que antes he contado, conviene ser muy prudentes frente a ese tipo de portentos documentales que cierta tradición nos ofrece como un híbrido entre testigo ideal y ventana mágica que permite contemplar sin sombras un pasado desaparecido. Aunque tales prodigios abundan en el terreno de lo mítico (como es el caso de la construcción nacional), por desgracia son absolutamente imposibles en el campo de las Ciencias Sociales o Humanas. Pasemos entonces a un proceso inverso: el desmontaje, la deconstrucción de la elegante y muy convincente fachada de la obra de Sahagún. Entremos ahora en la parte más oscura de su caja negra.

Edward Calneck (1974), investigador muy meticuloso, fue el primero en destacar que los textos indígenas de Sahagún, al ser contrastados con otras fuentes documentales, demostraban tener un carácter notablemente "sesgado y tendencioso" tanto en la exposición, como en la propia selección y jerarquización de aquellos datos que tenían o implicaban una significación sociológica. Aquello era debido, hoy lo sabemos bien, a los propios informantes indígenas utilizados por el franciscano, todos ellos procedentes de capas sociales nobiliarias o, al menos, privilegiadas. Informantes que cargaron las descripciones y hasta las meras alusiones a las capas sociales menos favorecidas, especialmente de artesanos y agricultores, con toda clase de prejuicios y desprecio jerárquico. En otras palabras, la mentalidad y la imagen de la sociedad indígena que refleja la variada información contenida en los textos en náhuatl de la Historia Universal corresponden a un sector o grupo social específico de la misma. Más aún, demuestra la existencia de conflictos internos entre las capas privilegiadas, así como entre grupos urbanos y étnicos distintos, como los pochtecha/tlatelolca frente a los teteuctin/tenochca...

Esta nueva visión notablemente matizada y fragmentada del mundo indígena terminó con las imágenes monolíticas previas; pero es que ha habido que agregar otro elemento de matización no menos importante,

\footnotetext{
${ }^{10}$ La idea fue característica de Ángel M. Garibay y su escuela, sirviendo de plataforma para abrir o potenciar todo un campo de estudios del que surgirían trabajos muy importantes, además de una serie significativamente titulada "Textos de los Informantes de Sahagún", que debía haber publicado los Códices Matritenses de la Historia Universal con su traducción al castellano. Desgraciadamente esta serie quedó trunca en su cuarto volumen (Sahagún 1958-1969).
} 
ni menos radical. A medida que se ha ido conociendo mejor el mundo indígena del período colonial, en el que - no lo olvidemos- hay que ubicar a los propios informantes de Sahagún, ha resultado indudable que "los nahuas", en palabras de Louise M. Burkhart (1989: 6), "reinterpretaron su propia cultura y su propio pasado a la luz de sus nuevas experiencias y necesidades; y que hasta su imagen de los antiguos aztecas fue en buena medida un producto colonial". De hecho, muchas ideas, obras y hasta géneros literarios que antes se consideraban típicamente prehispánicos, hoy los reconocemos como productos característicos de una sociedad sometida a procesos de aculturación muy intensos ${ }^{11}$.

Todas estas matizaciones afectan directamente a los materiales proporcionados por los ayudantes e informantes indígenas de Sahagún, negándoles su condición de "Voz prístina y desinteresada". Bien al contrario, esos materiales ahora son sólo comprensibles como un producto colonial, expresión de una capa social específica y de un grupo humano restringido. De hecho, las variantes - a veces muy importantes- que se han detectado entre textos sobre un mismo tema que se conservan en manuscritos correspondientes a distintas etapas de la obra de Sahagún, han adquirido una significación socio-cultural nueva que excede con mucho la puramente textual. Pero debe añadirse que tampoco Sahagún fue neutral ni en la selección, ni en el contenido de los distintos materiales que sus asistentes le fueron aportando. De hecho se han podido aislar casos muy significativos en los que el franciscano no dudó en intervenir, seleccionando una variable o incluso estableciendo una versión canónica (construida artificialmente), cuando las informaciones que le proporcionaban sus colaboradores eran discordantes, contradictorias o no compatibles entre $\mathrm{Si}^{12}$.

\footnotetext{
${ }^{11}$ En este sentido, el caso más llamativo y el que levantó una polémica más ácida fue el que planteó John Bierhorst (1985) con su singular, pero fascinante, edición e interpretación de los llamados Cantares Mexicanos, pieza literaria esencial en la restitución de una filosofía y una moral nahuas por parte de la escuela de Garibay y León-Portilla.

${ }^{12}$ El caso más claro y mejor conocido es el del calendario solar y su correlación con el europeo. Kubler y Gibson (1951: 52-54) observaron cómo en el mundo prehispánico existían varias correlaciones calendarias simultáneas, situación que se prolongó en el siglo XVI y que se refleja con mayor o menor consistencia en la cronística de la época. Sahagún resolvió este espinoso problema haciendo una junta con sus asistentes e informantes en la que fijó - de forma arbitraria- una sola correlación inamovible. Véase todo el proceso en Díaz Rubio y Bustamante (1983), a partir de la edición y traducción de la única carta conservada de las que dirigieron a Sahagún sus colaboradores indígenas. Para un estudio detallado sobre la cuestión calendárica en Sahagún, véase Bartl, Göbel y Prem (1989).
} 
Tales casos demuestran el papel activo que tuvo Sahagún en la elaboración del texto náhuatl y acreditan que el franciscano actuó no sólo como "colector" sino también como "editor"; pero todos ellos son casos extremos. Por eso, para poder continuar con nuestro proceso de análisis, importa mucho destacar que existe otro nivel de intervención por parte de Sahagún que sí es constante y general a toda la obra, de hecho es el que la configura y le da origen en cuanto "corpus" escrito.

Como se dijo antes, toda esa colección de textos en náhuatl fue recopilada a partir de una serie de cuestionarios metódicamente diseñados por fray Bernardino de Sahagún. Esos cuestionarios —omnipresentes a lo largo de toda la obra- son los que predeterminan sus características y contenidos. Tanta es su importancia que, aunque no se conserven de hecho, han podido ser reconstruidos con bastante detalle a partir de la propia colección textual en náhuatl, que es en realidad el conjunto de las respuestas conservadas ${ }^{13}$.

El análisis de los diferentes textos existentes permite precisar que, a medida que avanzaba la confección de la obra, los cuestionarios se fueron ampliando, enriqueciendo y matizando. En algunas ocasiones, al profundizar sobre ciertos temas, los cuestionarios alcanzaron una notable flexibilidad, lo que se refleja claramente en el tipo de respuestas y en su mayor calidad. Sin embargo, también es observable que el conjunto mismo de los cuestionarios básicos y, sobre todo, su articulación global (que corresponde a la propia idea rectora del proyecto de investigación) permanecieron inmutables. De esta manera, esos cuestionarios han dado origen a una riquísima colección textual escrita en lengua náhuatl y que procura ser la expresión de la mentalidad indígena; pero no hay que pasar por alto que las preguntas de estos mismos cuestionarios son las que han seleccionado los temas que debían incluirse en esa colección, más aún son las que han puesto límites a esos mismos temas y las que los han aislado o puesto en relación unos con otros. Los cuestionarios son tan determinantes que a través de sus preguntas se ha obligado inconscientemente a los informantes a que proporcionen explicaciones o definiciones de elementos de su cultura en unos términos morales o causales que les eran ajenos por completo. A ello se debe que en la Historia Universal haya libros cuya temática ha sido abordada con acierto, pero también que haya otros - fallidos - cuya temática apenas ha podido ser captada o planteada. El propio Sahagún era muy consciente de esas deficiencias, aunque no supiera cuál era la razón metodológica de las mismas.

13 Véase su estudio y reconstrucción en Alfredo López-Austin (1974), que sigue siendo el trabajo de referencia básico. 
Ejemplos claros de libros que Sahagún consideraba fallidos son el Libro III y el Libro VII del Códice Florentino. En el Libro III pretendía estudiar lo que podríamos llamar "geografía metafísica o celeste", es decir los cielos y los infiernos, los lugares donde vivían los dioses y a los que iban los muertos. El objetivo era obtener una descripción de la vida ultraterrena según su caracterización moral (buenos-dichosos-premiados y malos-desgraciados-castigados). Pero para los antiguos nahuas cielos e infiernos eran una cuestión puramente cosmográfica y los lugares a los que iban los muertos tenían que ver con la forma en que se producía físicamente el fallecimiento de la persona, sin connotación moral alguna. De esta manera los cuestionarios ahondaron en un campo para el que no había respuesta posible y éstas fueron escasas, incompletas e insatisfactorias. Sahagún trató de arreglarlo de diferentes maneras, pero sin conseguirlo. En consecuencia, la cosmografía y cosmología mexicanas están ausentes de la obra; en su lugar, el franciscano puso diferentes mitos sobre el origen de los dioses, aunque con resultado igualmente fallido e incompleto, porque una vez más Sahagún interpretaba la mitología según la teoría moral dominante en su época. Lo mismo ocurre, pero en sentido inverso, con el libro VII, cuya temática es la "astrología natural". La diferenciación entre astrología natural y judiciaria era muy importante en la Europa renacentista, pero totalmente ajena para la América prehispánica. De nuevo Sahagún utilizaba un cuestionario al que los indígenas difícilmente podían responder y, una vez más, fue imposible recoger la imagen del cosmos que tenían los mexicanos.

Es en este nivel determinante, pero subyacente e implícito a los textos mismos, donde se hace central la figura de Sahagún, con sus ideas, prejuicios e intenciones. El franciscano, visto desde esta perspectiva, actúa como "autor" sobre los textos en náhuatl porque, aunque es verdad que la relación entre los informantes y el investigador fue directa, también es cierto que esa relación estuvo mediatizada, más aún, estuvo articulada y condicionada a través de cuestionarios predeterminados y preestablecidos. Por eso se produce, hablando en términos generales, que los textos derivados de las entrevistas hayan sido categorizados de entrada por el propio investigador y no por sus informantes.

La Antropología tiene una larga experiencia sobre estas cuestiones metodológicas y yo no voy a entrar ahora en mayores detalles. Pero sí me importa destacar que los textos obtenidos mediante este sistema, a pesar de sus claros inconvenientes, ofrecen como elementos positivos una gran concisión y riqueza informativa, así como una gran facilidad para la articulación de esa información en modelos generales o comparativos. 
Precisamente tales elementos fueron objetivos prioritarios y metódicamente buscados por Sahagún, y esto está en relación directa con la tercera de las características principales de su Historia Universal, característica que no es menos importante que las otras dos que se han mencionado anteriormente.

La Historia Universal, empezando por esa especialísima colección textual en náhuatl que constituye su núcleo, está cuidadosamente organizada a partir de un sistema clasificatorio que se pretende - no por casualidad- "universal". Desde su mismo principio, al elaborarse los cuestionarios, la obra fue diseñada y después reorganizada y matizada a partir de un modelo articulador del conocimiento que ordena jerárquicamente a todas las criaturas del Universo en una larga cadena: de Dios (o los dioses, en este caso) al hombre; del hombre a los animales, plantas y minerales. Ese modelo proporciona a la obra un estilo enciclopédico tan acentuado y característico que, con frecuencia, la Historia Universal ha sido definida como una verdadera "enciclopedia náhuatl" o "de la cultura de los antiguos nahuas"14. Más aún, el reconocimiento de tal característica ha llevado incluso a buscar y señalar los posibles antecedentes clásicos y medievales de la obra o, más simplemente, a inscribirla —en palabras de Alfredo López-Austin (1974: 120) - dentro de "una línea del pensamiento humano en continua evolución, que se origina en los estudios zoológicos griegos, pasa por las historias naturales latinas y llega al Nuevo Mundo en la forma de enciclopedias medievales en las que se recogen todos los seres en un riguroso orden jerárquico, empezando por la Trinidad y terminando con los minerales".

De esta manera resulta que la Historia Universal de las Cosas de Nueva España nos presenta la cultura de los antiguos mexicanos y la forma misma en que éstos veían su mundo adaptada y seleccionada según un modelo clasificador en el que es fácil reconocer una tradición europea de origen clásico. Más áun, se trata en realidad de todo un complejo de ideas, aquél que Arthur O. Lovejoy (1964) llamó "the great chain of being", demostrando que no sólo implicaba una ordenación del Universo, sino también un cierto tipo de relaciones entre los seres, un modo de conocimiento, un papel para Dios en ese cosmos y un lugar y destino para el hombre.

${ }^{14}$ Como en tantas otras ocasiones, fue Ángel Garibay el primero en señalar que la obra de Sahagún tenía las características de una "enciclopedia de la cultura de los nahuas de Tenochtitlan" (Garibay 1953-54:2, 64). Pero se debe a Donald Robertson el descubrimiento de que era una verdera "Mexican Enciclopedia" que, "so far as the organization of the content is concerned, belongs in the tradition of medieval enciclopedias" (Robertson 1966; 1959: 169-172, cita en p. 171). 
La ordenación que tanto caracteriza a la Historia Universal no se reduce pues a una simple cuestión formal, sino que es expresión de un modo de pensar e indagar que pretende alcanzar un conocimiento cierto y completo, de ahí la condición "enciclopédica", en sentido literal, de la obra. A ello alude también el título mismo que Sahagún le impuso. "Historia" aparece aquí no en el sentido de crónica o anales, sino en el más genérico de "descripción que se hace de las cosas", cualquiera que sea su naturaleza o condición ${ }^{15}$. En cuanto al adjetivo "Universal", Sebastián de Covarrubias lo definía todavía en 1611 como alusivo a "el que tiene noticia de cosas diferentes y babla en ellas científicamente", entendiéndose por "ciencia", "el conocimiento cierto de alguna cosa por su causa"16. Así pues, con "Historia Universal" Sahagún quería decir "descripción que tiene noticia de muchas cosas diferentes y habla de ellas científicamente".

Estas últimas observaciones no responden a una simple preocupación crítica y erudita de restituir a la obra de Sahagún el título que él le dio, frente al casi equivalente de Historia General que una larga tradición editorial ha consagrado desde el siglo XIX. La insistencia en el término "universal" se basa en que con él Sahagún quiso dejar explícita, desde el mismo título, una última característica esencial de su obra: su condición científica, el rigor admirable con el que está hecha, la autorización y certeza de su contenido.

Ésta es una característica común a todo el proyecto y que lo define desde el principio, pero que se dirige particularmente al texto básico en lengua náhuatl, que es precisamente el que Sahagún está más interesado en autorizar. A esa característica, que es muy perceptible para los investigadores actuales, corresponde también la profunda preocupación metodológica que Sahagún expresa:

Todos los escriptores trabaxan de autorizar sus escripturas lo mejor que pueden, unos con testigos fidedignos, otros con otros escriptores que ante dellos han escripto, los testimonios de los cuales son habidos por ciertos; otros con testimonios de la Sagrada Escriptura. A mí me han faltado todos estos fundamentos para autorizar lo que en estos doce libros tengo escripto, y no hallo otro fundamento para autorizarlo sino poner aquí la relación de la diligencia que hice para saber la verdad de todo lo que en estos libros he escripto (Sahagún 1988: 77).

Así comienza, por ejemplo, el conocidísimo "prólogo" al Libro II del Códice Florentino, un texto magistral en el que Sahagún describe la forma en que reunió sus materiales y compuso su obra. Modo muy científi-

\footnotetext{
15 Diccionario de Autoridades (1984: s.v).

${ }^{16}$ Covarrubias (1984: s.v).
} 
co el adoptado por el franciscano, al autorizar y certificar los resultados de una investigación primaria a partir del método mismo seguido en su elaboración. Por desgracia, Sahagún no fue igualmente explícito a la hora de contarnos los modelos precisos que empleó y las categorías que utilizó para seleccionar y organizar en su obra esos mismo materiales que con tanto cuidado nos dice cómo reunió. Por supuesto que tales modelos concretos existieron y a su definición yo mismo le he dedicado bastantes páginas que ahora no vienen al caso. Lo que importa ahora es que una vez más certifican la presencia activa y condicionadora, hasta en los detalles más nimios, de la figura de Sahagún sobre su obra, tanto en náhuatl como en castellano, a pesar de que por su estilo tan científicamente depurado parezca que está ausente.

Llegamos así al final de nuestro recorrido, un recorrido en el que he intentado llamar la atención sobre el carácter inmanente, condicionado histórica y socialmente, de toda fuente de información, sea ésta escrita u oral, sea antigua o más reciente.

El caso de Sahagún, que he seleccionado no sólo por una cuestión afectiva y personal, me parece ejemplar en muchos sentidos. En primer lugar, es un modelo paradigmático de cómo unas mismas características pueden ser interpretadas como las mayores virtudes y -al mismo tiempo- los mayores defectos de una misma obra, o encadenar en secuencia las unas con los otros. Pero además se trata de una de las pocas obras que claramente exige para su estudio y utilización considerarla integradamente desde una multitud de ópticas: teniendo en cuenta a los informantes como autor (y las consecuencias que ello implica), al colector como autor (y las nuevas consecuencias de esa perspectiva) y, por supuesto, a los lectores mismos como autor, porque ellos son los que restituyen en cada ocasión la obra escrita, al leerla desde sus propias ópticas, sus propios problemas y sus propios referentes. Esa variación en las lecturas, a lo largo de los años, es lo que explica la variación misma en la apreciación de la figura y la obra de Sahagún, en la forma misma en que consideramos la historia del México Central prehispánico y colonial..., y es la que explica que continuamente podamos releer y aprovechar unos textos que - precisamente por eso- llamamos "clásicos".

\section{BIBLIOGRAFÍA CITADA}

Bartl, Renate; Barbara Göbel y Hans Prem. 1989. "Los calendarios aztecas de Sahagún". Estudios de Cultura Nábualt 19: 13-82.

BiERHORST, JoHn. 1985. "General Introduction", en J. Bierhorst (ed.), Cantares Mexicanos. Songs of the Aztecs: 1-122. Stanford: Stanford University Press. 
BROWNE, WALDEN. 2000. Sabagún and the Transition to Modernity. Norman: University of Oklahoma Press.

BurkHART, LuISE M. 1989. The Slippery Earth. Nabua-Christian Moral Dialogue in Sixteentb-Century. Tucson: The University of Arizona Press.

Bustamante GARCía, Jesús. 1989. La obra etnográfica y lingüística de fray Bernardino de Sabagún. Madrid: Universidad Complutense.

- 1990. Fray Bernardino de Sabagún: una revisión crítica de los manuscritos y de su proceso de composición. México: UNAM.

- 1992. "Retórica, traducción y responsabilidad histórica: claves humanísticas en la obra de Bernardino de Sahagún", en B. Ares, J. Bustamante, F. Castilla y F. del Pino, Humanismo y visión del otro en la España moderna: 243-375. Madrid: CSIC.

CALNECK, EDWARD E. 1974. "The Sahagún Text as a source of sociological information", en Edmonson (ed.), Sixtennth-Century Mexico. The Work of Sabagun: 179-204. Alburquerque, Nuevo México: School for American Research Press.

COVARRUBIAS, SEBASTIÁN DE. 1984. Tesoro de la lengua castellana o española. Primer Diccionario de la Lengua (1611). Madrid-México: Turner.

Díaz Rubio, Elena y Jesús Bustamante García. 1983. "Carta de Pedro de San Buenaventura a fray Bernardino de Sahagún acerca del calendario solar mexicano". Revista Española de Antropologia Americana 13: 109-120.

Edmonson, Munro E. (ed.). 1974. Sixteenth-Century Mexico. The Work of Sabagun. Alburquerque: School of American Research-University of New Mexico.

GARIBAY, ÁNGEL M. ${ }^{a}$. 1953-54. Historia de la literatura Nátbuatl. México: Porrúa.

KLOR DE AlvA, J. JORGE; HENRI B. NiChOlSON y ElOISE QUIÑONES KeBER (eds.). 1988. The Work of Bernardino de Sabagún. Pioneer Ethnographer of Sixteenth-Century Aztez Mexico. Albany: Institute for Mesoamerican Studies-The University at Albany State University of New York.

Kubler, GeORge y CHARLES GiBSON. 1951. The Tovar Calendar. New Haven: Memoirs of the Connecticut Academy of Arts and Sciences, vol. XI.

LEÓN-Portilla, Miguel. 1987. Bernardino de Sabagún. Madrid: Historia 16-Quorum.

LÓPEZ-AuSTIN, ALFREDO. 1974. "The Research Method of Fray Bernardino de Sahagun: The Questionnaires", en Edmonson (ed.), Sixteenth-Century Mexico. The Work of Sabagun: 111-149. Alburquerque: School of American Research-University of New Mexico.

Lovejoy, Arthur O. 1964. The Great Chain of Being. A Study of the History of an Idea. Cambridge, Massachussetts: Harvard University Press.

Nicolau D'Olwer, LuIs. 1952. Fray Bernardino de Sabagún (1499-1590). México: Instituto Panamericano de Geografía e Historia.

ROBERTSON, DONALD. 1959. Mexican Manuscript Painting of the Early Colonial Period: The Metropolitan Schools. New Haven: Yale University Press.

- 1966. "The Sixteenth Century Mexican Encyclopedia of Fray Bernardino de Sahagun". Cabiers d'Histoire Mondiale 9 (3): 617-628.

SAHAGÚN, FraY BERnARDINO DE. 1942. "Breve Compendio de los ritos idolátricos de Nueva España" (edición, notas y comentarios del P. Livario Oliger). Antonianum 17: $3-38$ y $133-174$.

- 1950-80. Florentine Codex. General History of the Things od New Spain (edición y traducción del texto náthuatl por Arthur J. O. Anderson y Charles E. Dibble). Santa Fe, Nuevo México: School of American Research-The University of Utah.

- 1958-69. Textos de los informantes de Sabagún. Vol. I: "Ritos, sacerdotes y atavíos de los dioses" (ed. y trad. Miguel-León Portilla), 1958; vol. II: "Veinte Himnos sa- 
cros de los nahuas" (ed. y trad. Ángel M. Garibay), 1958; vol. III: "Vida económica de Tenochtitlan. Pochtecayotl, arte de traficar" (ed. y trad. de Ángel M. Garibay), 1961; vol. IV: "Augurios y abusiones" (ed. y trad. de Alfredo López-Austin), 1969. México: UNAM.

- 1970. Códice Florentino. Historia general de las cosas de la Nueva España. Manuscrito 218-220 de la Colección Palatina de la Biblioteca Medicea-Laurenziana (edición facsimilar). Florencia/México: Casa Editorial Giunti Barbera/Archivo General de la Nación.

- 1988. Historia general de las cosas de Nueva España (primera edición del texto castellano del Códice Florentino, por Alfredo López-Austin y Josefina García Quintana). Madrid: Alianza.

- 1990. Breve compendio de los ritos idolátricos que los indios de esta Nueva España usaban en tiempo de su infidelidad (edición facsimilar, con estudio, transcripción y notas de M. ${ }^{a}$ Guadalupe Bosch de Souza). México: Ediciones Lince.

WOOLGAR, STEVE. 1988. Science: the Very Idea. Londres: Routledge.

- 1991. Ciencia: Abriendo la caja negra. Barcelona: Anthropos. 